\title{
Cell Analysis
}

Volume 1 
A Continuation Order Plan is available for this series. A continuation order will bring delivery of each new volume immediately upon publication. Volumes are billed only upon actual shipment. For further information please contact the publisher. 


\section{Cell Analysis}

\section{Volume 1}

Edited by

NICHOLAS CATSIMPOOLAS

Boston University School of Medicine

Boston, Massachusetts 
Main entry under title:

Cell analysis.

Bibliography: $\mathrm{p}$.

Includes index.

1. Cytology-Technique. 2. Cells-Analysis. I. Catsimpoolas, Nicholas. [DNLM: 1 . Cells-Analysis-Periodical. W1 CE126H]

QH585.C44

$574.87^{\prime} 028$

$82-5289$

ISBN 978-1-4684-4099-7

ISBN 978-1-4684-4097-3 (eBook)

AACR2

DOI 10.1007/978-1-4684-4097-3

(C) 1982 Plenum Press, New York

Softcover reprint of the hardcover 1st edition 1982

A Division of Plenum Publishing Corporation

233 Spring Street, New York, N.Y. 10013

All rights reserved

No part of this book may be reproduced, stored in a retrieval system, or transmitted in any form or by any means, electronic, mechanical, photocopying, microfilming, recording, or otherwise, without written permission from the Publisher 


\section{Contributors}

R. B. Allan, Department of Cell Biology, University of Glasgow, Glasgow G12 $8 Q Q, U . K$.

James W. Bacus, Medical Automation Research Unit, Rush-Presbyterian-St. Luke's Medical Center, Chicago, Illinois 60612

S. A. Ben-Sasson, The Hubert H. Humphrey Centre for Experimental Medicine and Cancer Research. The Hebrew University-Hadassah Medical School, Jerusalem, Israel

Michael W. Berns, Department of Developmental and Cell Biology, University of California, Irvine, Irvine, California 92717

Francis Dumont, Unité de Cancérologie Expérimentale et de Radiobiologie, INSERM U95, 54500 Vandoeuvre-les-Nancy, France. Present address: Department of Immunology, Merck, Sharp and Dohme Research Laboratories, Rahway, New Jersey 07065

N. B. Grover, The Hubert H. Humphrey Centre for Experimental Medicine and Cancer Research, The Hebrew University-Hadassah Medical School, Jerusalem, Israel

Volker Kachel, Max-Planck-Institut für Biochemie, D-8033 Martinsried, West Germany

J. M. Lackie, Department of Cell Biology, University of Glasgow, Glasgow G12 8QQ, U.K.

J. Naaman, The Hubert H. Humphrey Centre for Experimental Medicine and Cancer Research, The Hebrew University-Hadassah Medical School, Jerusalem, Israel

Gary C. Salzman, Life Sciences Division, Los Alamos National Laboratory, University of California, Los Alamos, New Mexico 87545

Robert J. Walter, Department of Developmental and Cell Biology, University of California, Irvine, Irvine, California 92717

P. C. Wilkinson, Department of Bacteriology and Immunology, Western Infirmary, University of Glasgow, Glasgow G11 6NT, U.K. 


\section{Preface}

The selective combination of physical, biochemical, and immunological principles, along with new knowledge concerning the biology of cells and advancements in engineering and computer sciences, has made possible the emergence of highly sophisticated and powerful methods for the analysis of cells and their constituents. This series on Cell Analysis is, therefore, aiming at providing the theoretical and practical background on how these methods work and what kind of information can be obtained. Cell Analysis will cover techniques on cell separation, cell identification and classification, characterization of organized cellular components, functional properties of cells, and cell interactions. Applications in cell biology, immunology, genetics, toxicology, specific diseases, diagnostics and therapeutics, and other areas will be covered whenever relevant results exist.

Nicholas Catsimpoolas

Boston, Massachusetts 


\section{Contents}

Chapter 1

Quantification of Red Blood Cell Morphology

James W. Bacus

I. History. . . . . . . . . . . . . . . . . . . . . . . 1

II. Details of Red Cell Measurements .................. 3

III. Cell Sample Population Distributions ................. 11

IV. Discussion and Summary . . . . . . . . . . . . . . . . . . 25

References................................. 30

Chapter 2

Laser Microirradiation and Computer Video Optical Microscopy in Cell Analysis

Michael W. Berns and Robert J. Walter

I. Introduction ............................ 33

II. Laser Microbeams ........................... 34

III. Computer-Enhanced Video Microscopy for Laser Microsurgery ................................. $\quad 35$

A. Contrast Enhancement ..................... $\quad 37$

B. Edge Enhancement ........................ 37

C. Pseudocolor Enhancement .................... $\quad 40$

IV. Chromosome Microsurgery . . . . . . . . . . . . . . . $\quad 40$

A. Mitotic Organelles ......................... 43

B. Cytoplasm ............................. 48

V. Plant Cell Development (Chloroplast Irradiation) $\ldots \ldots \ldots . \quad 49$

VI. Developmental Cellular Neurobiology . . . . . . . . . . . . . . 49

VII. Pattern Formation . . . . . . . . . . . . . . . . . . . . . . 50

VIII. Conclusions .............................. 51

References............................. 51 
Chapter 3

Combination of Two Physical Parameters for the Identification and Separation of Lymphocyte Subsets

\section{Francis Dumont}

I. Introduction .............................. 55

II. Methods . . . . . . . . . . . . . . . . . . . . . . . . 56

A. Preparation of Cell Suspensions ............... 56

B. Fractionation of Cell Suspensions by Free-Flow Preparative Electrophoresis ............................ 57

C. Separation of Cells by Isopycnic Centrifugation in a Percoll Gradient ................................ 60

D. Cell Enumeration and Size Distribution Analysis ....... 61

E. Construction of the Fingerprints .................. 63

F. Interpretation of the Fingerprints ............... 65

III. Applications .................................. 66

A. Fingerprint Analysis of Murine Thymocytes .......... 66

B. Fingerprint Analysis of Murine Splenocytes .......... 75

C. Fingerprint Analysis of Murine Lymph Node Cells ..... 84

IV. Conclusion ............................... $\quad 87$

References............................... 87

Chapter 4

\section{Electrical Sizing of Cells in Suspension}

N. B. Grover, S. A. Ben-Sasson, and J. Naaman

I. Introduction . . . . . . . . . . . . . . . . . . . . . . 93

II. Theory . . . . . . . . . . . . . . . . . . . . . . . . . 94

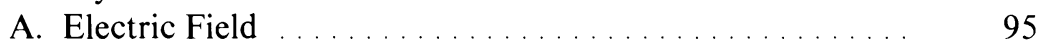

B. Hydrodynamic Field . . . . . . . . . . . . . . . . . 96

C. Cell Volume ... . . . . . . . . . . . . . . . . . . . . . . 96

D. Shape and Orientation . . . . . . . . . . . . . . . . . 97

E. Membrane Conductance ........................ . 98

F. Electrical Size . . . . . . . . . . . . . . . . . . . . . . . . . . . . 99

III. Instrumentation . . . . . . . . . . . . . . . . . . . . . 99

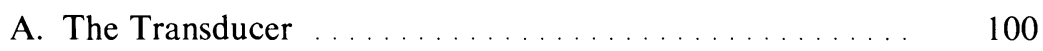

B. The Amplifiers ......................... 100

C. The Selector .......................... 100

D. Data Handling ........................... 102

E. Auxiliary Units .......................... 102 


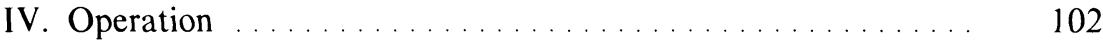

A. Cell Suspensions . . . . . . . . . . . . . . . . . . . 102

B. Orifice Dimensions . . . . . . . . . . . . . . . . . . . . . . . 103

C. Amplification ........................... 103

D. Calibration .......................... 104

V. Applications .............................. 104

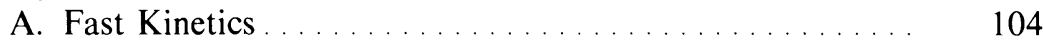

B. High Fields ........................... 105

C. Membrane Permeability ..................... 105

VI. The Future .............................. 106

A. Sensitivity ........................... 106

B. Multiparameter Systems ..................... 107

References.............................. 107

Chapter 5

\section{Light Scattering Analysis of Single Cells}

Gary C. Salzman

I. Introduction .......................... 111

II. Theoretical Considerations ..................... 112

A. Scattering Theory ....................... 112

B. Coated Sphere Model Tests ...................... 113

C. Expected Detector Responses ................... 113

III. Recent Applications of Forward and $90^{\circ}$ Scatter ......... 124

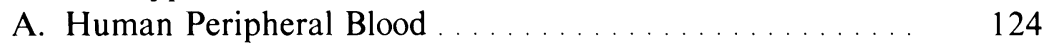

B. Osmolarity Effects ......................... 125

C. Hematopoietic Stem Cells . . . . . . . . . . . . . . . . 126

D. T-Lymphocyte Subclasses . . . . . . . . . . . . . . . . 128

E. Non-Hodgkin's Lymphomas .................... 129

F. Cervical Cell Analysis with $90^{\circ}$ Scatter . . . . . . . . . . 129

G. Sputum Samples . . . . . . . . . . . . . . . . . . . . . . . . 129

IV. Multiangle and Multiwavelength Scattering ........... 130

A. Multiangle Scattering ..................... $\quad 130$

B. Two-Color Light Scattering . . . . . . . . . . . . 133

V. Fourier Transform Techniques .................. 134

VI. Polarization Studies-New Directions ................ 136

A. Polarization Transformations ................. 136

B. Circular Intensity Differential Scattering .......... 137

VII. Conclusion . . . . . . . . . . . . . . . . . . . . . . 138

References............................... 138 
Chapter 6

\section{Methods for Measuring Leukocyte Locomotion}

P. C. Wilkinson, J. M. Lackie, and R. B. Allan

I. Introduction ............................. 145

II. Visual Assays . . . . . . . . . . . . . . . . . . . . . 151

A. Time-Lapse Filming ...................... 151

B. Indirect (Non-Time-Lapse) Methods . . . . . . . . . . . 161

C. Analysis of Cell Movement ....................... 162

D. The Orientation Assay . . . . . . . . . . . . . . . . . . . . . 165

E. The Candida Assay .......................... 168

III. The Micropore Filter Assay . . . . . . . . . . . . . . . . . 171

A. Principle .......................... 171

B. Apparatus . . . . . . . . . . . . . . . . . . . . . . . 171

C. Measurement of Leukocyte Locomotion in Filter Assays . . 174

D. The Checkerboard Assay . . . . . . . . . . . . . . . . . . . 179

E. Automated Method ......................... 181

IV. The Under-Agarose Assay . . . . . . . . . . . . . . . . . . . . 182

V. Miscellaneous Techniques ...................... 184

A. The Capillary-Tube Assay for Macrophage Locomotion . . 184

B. The Agarose Microdroplet Assay . . . . . . . . . . . . . . . 186

VI. Factors Influencing Locomotion . . . . . . . . . . . . . . . . 187

A. Cell-Substratum Interactions . . . . . . . . . . . . . 187

B. Composition of the Fluid Phase . . . . . . . . . . . . 188

C. Cell-Cell Interactions . . . . . . . . . . . . . . . . . . . . . 189

References..... . . . . . . . . . . . . . . . . . . . . . . . . 190

Chapter 7

Sizing of Cells by the Electrical Resistance Pulse Technique: Methodology and Application in Cytometric Systems

Volker Kachel

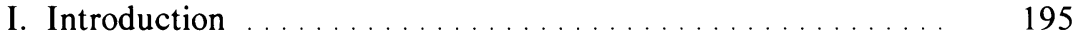

A. Reasons for Measuring Size of Biological Cells ....... 195

B. A Short Review on the History of Electrical Sizing ..... 196

II. Basic Events and Problems in Electrically Sizing Transducers 198

A. The Basic Coulter Effect . . . . . . . . . . . . . . . . . . 198

B. Fundamental Relations of Particle Volume, Electrical Resistivity, Resistance Change, and Pulse Height ...... 199 
C. Flow Conditions and Flow Line Coordination in the Sensitive Zone .............................. 217

D. Behavior of Biological Particles in the Sizing Transducer 224

III. Instrumentation . . . . . . . . . . . . . . . . . . . 248

A. General Conditions and Review ............... 248

B. Transducer . . . . . . . . . . . . . . . . . . . . . . . . . . 249

C. Electronic Instrumentation ..................... 254

D. Electronic Pulse Handling Devices as Alternatives to Hydrodynamic Focusing ..................... 263

IV. Calibration Problems of Electrical Sizing Flow Cytometers... 264

A. Calibration in Long Orifices with Homogeneous Field .... 264

B. Calibration with Short Orifices ................ 274

C. Calibration with Reference Particles .............. 276

V. Applications of the Metricell Cell Volume Analyzer ....... 277

A. Erythrocyte Volume Distribution Curves of Young Rats .. 277

B. Red Blood Cell Aggregation in Humans ............ 278

C. Thrombocyte Volume Distributions Measured from Whole Blood

D. Effect of Nerve Growth Factor on Pheochromocytoma Cells

VI. Combination of Electrical Sizing with Multiparameter Optical Flow Analysis

A. The Fluorescence-Volume (FLUVO) Transducer ...... 281

B. Cell Volume-Cell Absorption Transducer . . . . . . . . . . 285

C. Electronic Evaluation ...................... $\quad 286$

D. Examples of Combined Electrical-Optical Cell Analysis . . 290

VII. Sorting Activated by Electrical Sizing . . . . . . . . . . . . . . . 297

VIII. Size-Triggered Imaging in Flow . . . . . . . . . . . . . . . 302

A. Instrumentation for Size-Triggered Imaging . . . . . . . . . 302

B. Problems of Visualization Cell Structures . . . . . . . . 306

C. Examples of Cell Imaging in Flow . . . . . . . . . . . 307

Appendix ................................. . . 313

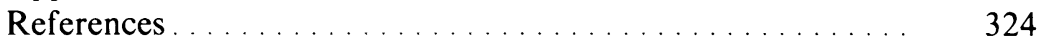

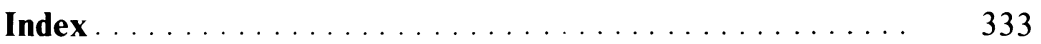

